\title{
Tongue coupling as a therapeutic strategy: electromyographic analysis of different training approaches
}

\author{
Loslene Goulart Souza1 \\ https://orcid.org/0000-0001-7858-8332 \\ Roberta Libindo de Figueiredo ${ }^{1}$ \\ https://orcid.org/0000-0002-8695-2664 \\ Yulieth Paulina Stave Gómez ${ }^{2}$ \\ https://orcid.org/0000-0002-5782-6917 \\ Guilherme Brasil ${ }^{3}$ \\ https://orcid.org/0000-0003-3486-642X \\ Fabiane Miron Stefani ${ }^{3}$ \\ https://orcid.org/0000-0002-8531-6594 \\ Angela Ruviaro Busanello-Stella ${ }^{1}$ \\ https://orcid.org/0000-0003-3144-5857
}

\begin{abstract}
Universidade Federal de Santa Maria UFSM, Santa Maria, Rio Grande do Sul, Brasil.

2 Universidade Federal de Santa Maria UFSM, Programa de Pós-Graduação em Distúrbios da Comunicação Humana, Santa Maria, Rio Grande do Sul, Brasil. ${ }^{3}$ Universidade Federal de Santa Catarina UFSC, Florianópolis, Santa Catarina, Brasil.
\end{abstract}

This project was carried out at the Universidade Federal de Santa Maria UFSM, Santa Maria, Rio Grande do Sul, Brazil.

Conflict of interests: Nonexistent

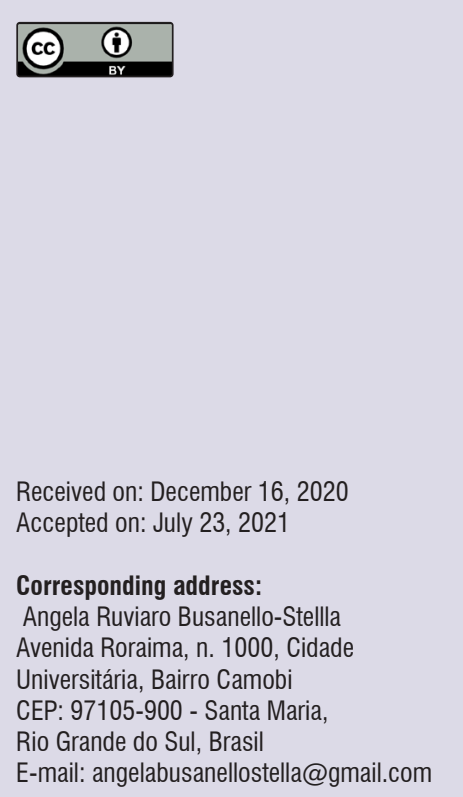

\section{ABSTRACT}

Purpose: to compare the effect of two tongue coupling approaches on the electrical activity of the suprahyoid musculature in young women.

Methods: the sample comprised 47 women aged 18 to 27 years, distributed into groups, according to exercise training time -10 and 15 seconds. They answered an initial questionnaire and were submitted to speech-language-hearing clinical assessment, body mass index calculation, and electromyographic assessment. Two electrodes were positioned bilaterally on the skin in the region of the suprahyoid musculature to pick up the electromyographic signal. The women performed the tests with resting periods and maximum voluntary contraction, coupling the tongue with the hard palate as strongly as possible, followed by three training series coupling the tongue with the palate. The collected data regarding amplitude and frequency of the myoelectrical activity were analyzed, by comparing the performance within the two groups and one another with the Friedman and One-Way ANOVA tests, and Mann-Whitney U test, at the $5 \%$ significance level.

Results: no statistical significance was observed between the groups when comparing their performance in the series, neither was there any decrease in the median frequency in each group while performing the three series.

Conclusion: no effect on the electrical activity of the suprahyoid musculature was found in young women when they performed isometric tongue coupling exercise training.

Keywords: Tongue; Electromyography; Stomatognathic System; Myofunctional Therapy 


\section{INTRODUCTION}

Tongue coupling (TC) is an exercise employed in the myofunctional rehabilitation process ${ }^{1}$, in which the tip and body of the tongue are coupled against the palate, keeping the resistance for a given time ${ }^{2}$.

The objective of this isometric exercise is to increase muscle strength. It is performed more slowly, holding a steady static contraction in the full length of the muscle fiber $^{3}$. It is made possible by the combined effort of the tongue extrinsic (genioglossus, styloglossus, palatoglossus, and hyoglossus) and intrinsic musculature (superior longitudinal, inferior longitudinal, vertical, and transverse). The first enables the tongue to move in all directions, whereas the second is responsible for changing the shape of this organ into tapered, flattened, folded, and so on ${ }^{4,5}$. There is also evidence of the elevation of the floor of the mouth while sucking the tongue against the palate, showing the active participation of the suprahyoid musculature when performing this exercise ${ }^{6}$, though the main musculature is not in action?.

Although some studies in the literature use TC as a therapeutic strategy to treat obstructive sleep apneahypopnea syndrome ${ }^{8-10}$, mouth-breathing syndrome ${ }^{11}$, swallowing in older adults ${ }^{12}$, and so forth, most of them address its use in dysphagia ${ }^{13-16}$.

Indeed, the suprahyoid musculature plays an important role in swallowing, as it is related to laryngeal elevation. A study observed a relationship between the electrical activity of the suprahyoid musculature and tongue pressure when swallowing ${ }^{12}$, suggesting that TC with the palate can strengthen not only the intrinsic tongue musculature system but also the suprahyoid musculature. This muscle activity was approached in a study ${ }^{17}$, which used surface electromyography (SEMG) as a strategy to analyze the activity of the suprahyoid musculature and compare tongue strengthening exercises in dysphagia rehabilitation used in clinical practice.

SEMG is a resource used to quantitatively analyze muscle electrical activity, helping understand the muscle electrical activity patterns in the stomatognathic system in general ${ }^{18}$ and the suprahyoid musculature ${ }^{7}$. It is described in the literature as one of the technological resources used to complement clinical assessment, as well as to receive biofeedback in orofacial myofunctional disorder therapy ${ }^{19}$.

In this context, a Brazilian study verified the electrical activity of the suprahyoid musculature in healthy women with eight different isometric exercises for 5 seconds each, including TC. The study analyzed which one would be the most adequate as a reference to standardize the electromyographic signals. According to the authors, there was no difference in electrical activation between the various types of exercise ${ }^{20}$.

Another study investigated the correlation between tongue pressure measures and the electrical activity of the suprahyoid musculature. The volunteers had their maximum tongue pressure assessed with the lowa Oral Performance Instrument (IOPI) along with SEMG of the suprahyoid musculature. The tongue had to be pressed against the anterior and posterior hard palate with the IOPI for 2 and 10 seconds in the SEMG assessment. According to the authors, there is a moderate correlation between tongue pressure and the electrical potential of the muscle in question only when the posterior part of the tongue is used ${ }^{7}$.

Another potential use of SEMG is to analyze muscle fatigue, a common symptom in which the necessary or expected strength cannot be maintained for a set time ${ }^{21}$. The SEMG assesses the level of this symptom in static and dynamic contractions. Particularly in isometric contractions, the median frequency values decrease, while the signal amplitude increases, which is interpreted as the recruitment of motor units. Both indicate muscle fatigue ${ }^{22,23}$.

A crucial aspect in speech-language-hearing intervention, besides the abovementioned physiological issues and instrument potentials, is the time indicated for therapeutic strategies. There are various indications in the literature regarding the amount and frequency of isometric exercises. Some authors report 5 to 8 seconds in three to five series ${ }^{3} ; 5$ to 10 seconds in a single series ${ }^{24} ; 5$ to 30 seconds of sustained contraction in three series ${ }^{25}$; and 20 seconds in three series $^{4}$. Regarding TC, specifically, some studies use 5-second contractions adding up to 30-second sets, 5 days a week ${ }^{26}$, and 10 -second muscle contractions in three series, 2 days a week ${ }^{11}$. Since there is no specific standard for these aspects, and there is an evident lack of findings in the literature investigating $\mathrm{TC}$ as a single strategy, this topic should be studied more in-depth. This would help better define oral-motor function therapeutic procedures and better understand the physiology of the exercise employed.

Hence, the objective of this study was to compare the effect of two TC approaches on the electrical activity of the suprahyoid musculature in young women. 


\section{METHODS}

This research is an observational, descriptive, quantitative, cross-sectional study. It complied with the guidelines outlined in Resolution 466/2012 and was approved by the Research Ethics Committee of the Universidade Federal de Santa Maria (Federal University of Santa Maria - UFSM), Brazil, under evaluation report number 3.021.987.

The study sample comprised volunteers based on the following inclusion criteria: females, 18 to 30 years old, without speech-language-hearing complaints, able to perform TC. The volunteers with an abnormal body mass index (BMI); a history of speech-languagehearing therapy in the previous year, head and neck surgery, and/or tracheostomy; and signs suggestive of neurological or syndromic impairment were excluded from the study.

Sample homogeneity is an important factor; therefore, only women were maintained in the sample, so as to obtain similar physical characteristics and try to provide a scientific standard for this population.

As for their age, the findings in the literature showing that the muscle strength peaks at around 30 years old, were considered. It probably remains the same until 50 years old, when sarcopenia becomes sharper ${ }^{27}$.

The BMI - i.e., the person's weight in kilograms divided by their height squared - is a nutritional status indicator used in clinical practice ${ }^{28}$. It was one of the exclusion criteria because people with a high BMI can have more adipose tissue in the target region of the study and interfere with the electromyographic signal pick-up. As pointed out by the World Health Organization $(\mathrm{WHO})^{29}$, the $\mathrm{BMl}$ is highly sensitive to identify overweight and obese subjects. Its normal values range from 18.5 to $24.9^{28}$.

\section{Selection Procedures}

An initial questionnaire, a speech-language-hearing clinical assessment, and a BMI calculation to select the sample, were administered.

The initial questionnaire was administered to investigate the selection criteria and include or exclude the volunteers. The speech-language-hearing clinical assessment was conducted by a single researcher based on the clinical history and examination, aiming to investigate and complement the information in the questionnaire. The history was based on the MBGR protoco $^{30}$, and the stomatognathic system was assessed with the Orofacial Myofunctional Evaluation
Protocol with Scores (OMES) ${ }^{31}$, complemented with the MBGR protocol.

The participants had their weight and height measured respectively with a digital upright scale and a measuring tape to assess their $\mathrm{BM}^{28}$.

Having concluded the selection procedures, three participants were excluded for being overweight, totaling 47 participants. They were distributed into two groups - one with 10-second (G10) and the other, 15-second training series (G15). These time sets were chosen based on the previously mentioned divergence in the literature regarding time and frequency indications for the exercises $3,4,11,24-26$. Thus, it was chose to establish these training time sets because they were the average values found in the literature.

Given the scarcity of studies in subjects without specific pathologies, the inclusion of as many as possible, in each group, was aimed. However, there were discrepancies between them because of the impact of the COVID-19 pandemic, which interfered with the conclusion of data collection in the second group.

\section{Electromyographic Assessment and Muscle Training}

Data were collected with SEMG of the suprahyoid musculature and TC muscle training.

The electromyographic assessment was conducted by a single researcher, blinded to the previous assessments to avoid bias and differences in the collection procedure.

The participants were instructed about the examination process, got acquainted with the collection site and equipment, and were previously trained to check whether they would know how to properly perform the technique in the electromyographic procedures ${ }^{32}$. Then they sat comfortably on a chair, at 90 degrees, their feet slightly apart flat on the floor, hands relaxed on the legs, sitting up against the back of the chair, and the head upright. They were instructed to keep this posture throughout the exercises ${ }^{20}$.

The electrodes were placed on previously prepared sites, and the skin was cleaned, rubbing it with gauze damped with $70 \%$ alcohol. They were placed bilaterally on the skin, parallel to the anterior belly of the digastric muscle, a little below the mentalis muscle, and anteriorly to the neck - a position compatible with the region of the suprahyoid musculature ${ }^{12}$ (FIGURE 1a). Each participant was asked to press the tongue 
strongly against the hard palate, so as to achieve a better visualization of the region and locate the most prominent $\mathrm{area}^{7}$. A reference electrode (connected to the ground wire) was placed on the region of the sternum to avoid electromagnetic interference (FIGURE 1b). To pick up the electromyographic signal, active sensors with differential input manufactured by Miotec, connected to silver/silver chloride $(\mathrm{Ag} / \mathrm{AgCl})$ bipolar electrodes (manufactured by Miotec ${ }^{\circledR}$ ) were used. Care was taken to turn off any equipment that might interfere electromagnetically with the examination and keep them at a distance.

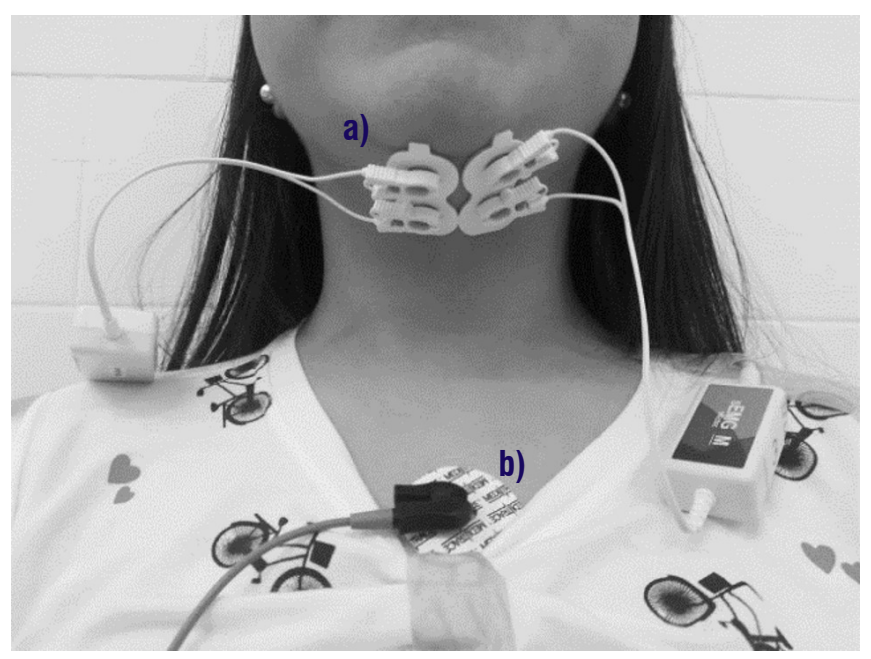

a) electrodes positioned in the region of the suprahyoid musculature.

b) reference electrode (ground)

Source: the authors

Figure 1. Placement of the electrodes over the suprahyoid musculature

Before collecting data on the muscle training, were realized (1) a 15-second rest and (2) maximum voluntary contraction (MVC), coupling the tongue at full strength for 5 seconds at a time ${ }^{33}$. The purpose of the rest was to analyze the quality of the signal and that of the MVC was to standardize the signal. In both, three collections were made in each situation, with an approximate 1-minute rest for muscle recovery in between collections ${ }^{34}$. The collection with the best signal quality of the three was chosen, according to the Fast Fourier Transform (FFT) frequency spectrum.

All the participants received the following instructions in the rest test before the collection: "Please, remain silent; you may close your eyes if you want but try not to swallow during the stipulated time". As for the MVC collection, the instruction was: "When I ask you, please couple the tongue as strong as possible, using a lot of strength on the tongue". As they performed it patients were encouraged to increase the tongue contraction against the palate: "Go, go, go".

The muscle training to compare the two situations, i.e., 10- and 15-second exercises, was recorded. These sets of time for each series based on the findings in the literature ${ }^{11,24-26}$ were chosen. Also, the recording of the training helped the researchers to control the exercises. The G10 performed three TC series with comfortable effort for 10 seconds, whereas the G15 performed TC in the same conditions but in three 15-second series. The exercises were performed in three series because, according to the literature, from the fourth series there may be a considerable increase in lactate production, causing great discomfort and fatigue ${ }^{35}$.

The electromyographic signal was collected and analyzed with the Miotool equipment (Miotec), with eight input channels, 14-bit resolution $A / D$ converter, and $20 \mathrm{~Hz}$ to $500 \mathrm{~Hz}$ band filters, as well as Miograph Suite Software. The amplitude $(\mu \mathrm{V})$ and signal frequency (MF), which were later standardized with the MVC were analyzed. The data were stored in a portable computer manufactured by Itautec S.A., powered by an Intel Pentium processor, and running Windows 7 Pro operating system, not connected to an electrical outlet to avoid its interference with the signal.

The statistical analysis was made in the Statistica 7.0 program, and the $5 \%(p<0.05)$ significance level was used. Initially, the Shapiro-Wilk test was used to verify the normal behavior of the variables. Most of the variables did not have a normal behavior; hence, the analysis with nonparametric tests was chosen. To compare the groups, the Mann-Whitney $U$ test was used; to analyze the exercise series with one another, the Friedman ANOVA test was applied. The variables related to MF analysis were the only ones with a normal behavior; thus, the analysis between the exercise series was made with the one-way ANOVA test.

\section{RESULTS}

The final sample comprised 47 volunteer women without speech-language-hearing complaints, distributed into two groups: 30 in G10 and 17 in G15. The age of those in $\mathrm{G} 10$ ranged from 18 to 27 years, mean of 22.7 years (272.8 months). In G15, the volunteers' age ranged from 19 to 26 years, mean of 22.6 years (267.17 months).

Most of the volunteers were speech-languagehearing pathology students. All of those in G10 were 
such students, while in G15 only four volunteers $(23.52 \%)$ were not from the speech-language-hearing sciences. Based on this characteristic, they were further divided according to clinical experience, whether they had any in oral-motor function. Those who had not yet taken oral-motor function practical courses were grouped with the ones who were not speech-languagehearing students, as they had no experience in the field.

Hence, G10 had 17 (56.66\%) volunteers without and $13(43.33 \%)$ with experience in the field. As for G15, there were $7(41.17 \%)$ without and 10 $(58.82 \%)$ with experience in the field. This distribution shows that, despite the sample having mostly speech-language-hearing pathology students, the groups had similar proportions of volunteers with and without clinical experience in oral-motor functions, ensuring the groups' homogeneity in this regard.

Table 1 describes the results of the comparison between the groups concerning the electrical activity (regarding amplitude) of the suprahyoid musculature, in each series, in both muscles, as well as the analysis throughout the three series within each group.

Table 2 describes the results of the analysis of the median frequency of the electrical activity of the suprahyoid musculature (regarding frequency) throughout the three series within each group.

Table 1. Distribution and analysis of the means and standard deviation of the standardized electrical activity values (regarding amplitude) of the suprahyoid musculature in both groups

\begin{tabular}{ccccc}
\hline \multirow{2}{*}{ Muscle/Series } & & G10 & G15 & \multirow{2}{*}{ p-value } \\
\cline { 3 - 4 } & & $\mathbf{X}($ SD) & $\mathbf{X}($ SD) & \\
\hline \multirow{3}{*}{ RSH } & Series 1 & $33.83(21.30)$ & $31.54(13.18)$ & $0.72 \mathrm{a}$ \\
& Series 2 & $36.03(24.87)$ & $33.99(14.40)$ & $0.73 \mathrm{a}$ \\
& Series 3 & $38.12(23.01)$ & $33.71(15.16)$ & $0.67 \mathrm{a}$ \\
& p-value & $0.64 \mathrm{~b}$ & $0.46 \mathrm{~b}$ & \\
\hline \multirow{3}{*}{ LSH } & Series 1 & $33.07(20.68)$ & $27.95(10.97)$ & $0.61 \mathrm{a}$ \\
& Series 2 & $34.00(21.03)$ & $30.40(13.08)$ & $0.67 \mathrm{a}$ \\
& Series 3 & $35.26(22.77)$ & $30.90(17.49)$ & $0.65 \mathrm{a}$ \\
& p-value & $0.96 \mathrm{~b}$ & $0.46 \mathrm{~b}$ & \\
\hline
\end{tabular}

Captions: RSH - right suprahyoid, LSH - left suprahyoid, G10 - group with 10-second tongue coupling training, G15 - group with 15-second tongue coupling training, $\mathrm{X}$ - mean, SD - standard deviation, a- analysis with Mann-Whitney U test, $\mathrm{b}$ - analysis with Friedman ANOVA test.

Table 2. Distribution and analysis of the means and standard deviation of the standardized values of the median frequency of the suprahyoid musculature per series of 10 and 15 seconds each

\begin{tabular}{cccc}
\hline \multirow{2}{*}{ Muscle/Series } & MF G10 & MF G15 \\
\cline { 2 - 4 } & Series 1 & $\mathbf{X}($ SD) & $\mathbf{X}$ (SD) \\
\hline \multirow{3}{*}{ RSH } & Series 2 & $176.51(24.39)$ & $156.73(17.96)$ \\
& Series 3 & $175.26(22.92)$ & $153.45(16.02)$ \\
& p-value & $175.50(23.43)$ & $152.22(16.77)$ \\
& Series 1 & $0.97 \mathrm{a}$ & $0.10 \mathrm{a}$ \\
\hline \multirow{3}{*}{ LSH } & Series 2 & $175.69(23.20)$ & $163.02(21.32)$ \\
& Series 3 & $175.97(19.02)$ & $158.99(21.48)$ \\
& p-value & $174.79(21.14)$ & $159.55(28.37)$ \\
\hline
\end{tabular}

Captions: RSH - right suprahyoid, LSH - left suprahyoid, MF - median frequency, X - mean, SD - standard deviation, a - One-Way ANOVA.

A second analysis to better assess how the clinical experience in oral-motor function influenced the results of this study was chosen. The same comparisons described above were made, but approaching the sample with 47 volunteers as distributed into groups with and without clinical experience in this field, 24 (51.06\%) without and 23 (48.93\%) with experience. There was no statistical significance in the comparison 
between the two types of participants regarding each exercise series or throughout the series. Thus, the volunteers' degree of experience was found not to be enough to influence the results of this study, and therefore will not be approached below.

\section{DISCUSSION}

Few studies have been carried out in healthy adult volunteers ${ }^{26}$, without neurogenic diseases and/or structural deficiencies in the oral cavity that might interfere with their oral-motor skills ${ }^{32}$.

A scarcity concerning the training choices was also noticed. A recent international study ${ }^{36}$, for instance, carried out a systematic review on the effects of isometric training on tongue strength and swallowing. All the studies they retrieved reported post-training strength gain in both healthy and dysphagic adults. However, their benefits to swallowing are not known for certain, neither has a standard protocol been established for isometric tongue strength training.

Brazilian studies have already reported this divergence, highlighting the need for trials approaching the physiological implications of exercises ${ }^{24,35}$. Therefore, this research was conducted with healthy volunteers and an investigation of immediate intervention, focusing on the effects of TC on the muscle fibers normally measured with SEMG.

No statistically significant difference was found between performing TC for 10 and 15 seconds when the SEMG data from the young women in this sample, who had no speech-language-hearing complaints were compared. No other study was found in the literature with this specific comparison. Hence, other possibilities found in the literature and their interrelationships with this study will be considered below.

Another study also used 10 -second TC at maximum strength as the only exercise ${ }^{16}$, but with 18 presbyphagic subjects. The authors found that the tongue pressure resistance training improved both the tongue strength and the hyoid musculature. It is important to point out, though, that the training approach had 5 series with rests in between exercises, taking place twice a day for 1 month.

Another interesting study ${ }^{20}$ compared the electrical activity of women's suprahyoid musculature, with 5 -second contractions in eight different tongue strength exercises, including TC. All the participants were submitted to clinical and electromyographic assessment, and no significant difference was found in muscle activation during the exercises.
Some studies conduct muscle training with controlled load levels to research which exercise condition would be most efficient. For instance, a Belgian study ${ }^{37}$ in healthy older adults carried out 8 weeks of training with IOPI. They were divided into three training groups, performing maximum isometric tongue pressure at different loads $-100 \%, 80 \%$, and $60 \%-$, besides a control group, which performed lip strengthening. Each session required 120 efforts (60 in anterior and 60 in posterior tongue regions), divided into 24 series with five 3-second repetitions, and a 30-second interval in between series. The authors found no significant difference between the groups but observed that lower loads had less training effect.

The previous study brings up an interesting issue regarding the intervention protocols proposed in the literature. It is scientifically crucial to control the exercise loads to try to understand in further detail the physiological muscle behavior. However, speech-languagehearing clinicians cannot carry out such control because it requires some type of instrument, such as SEMG, IOPI, and so forth, to provides biofeedback. In this sense, the present study aimed to carry out its investigation as close as possible to clinical conditions.

Broad training approaches, with various strategies, are also found in the literature ${ }^{13}$. The authors submitted 14 individuals with post-stroke tongue weakness to two protocols - one with tongue pressure training with swallowing tasks, and the other with tongue pressure and precision training without functional tasks. The tongue pressure resistance training had 24 sessions, administered two to three times a week, for 8 to 12 months. The results of this study showed a significant improvement in tongue strength, without residues in the vallecula after swallowing thin liquids, regardless of the treatment condition.

One observes that most of these studies have in common a great variability in therapeutic approaches. Moreover, none of them compared a given exercise performed in different time sets, focusing mainly on the effect of either of these time sets. Thus, the results of this study initially help verify that performing TC briefly in healthy women for either 10 or 15 seconds leads to no difference in terms of electrical activity.

An important aspect to consider when interpreting the results of the present study is the suprahyoid musculature's contribution to TC. The absence of statistical difference in the electromyographic data may be due to the target exercise, which dealt more strongly with the more intrinsic tongue muscles, such 
as the genioglossus, which might not be picked up with SEMG ${ }^{7}$.

As seen in Table 2, the electromyographic MF signal did not reveal any statistically significant difference throughout the exercise series in either group, neither did it show any decreasing behavior, which is characteristic of a possible muscle fatigue condition.

Even though the literature ${ }^{23}$ point to a higher incidence of muscle fatigue in isometric exercises, the absence of such phenomenon in the present study may be related to the time sets chosen for the static contractions. It is believed that 10 and 15 seconds of isometric exercises in the suprahyoid musculature of young women without speech-language-hearing complaint, even in three repetition series, do not deposit enough substrates inside the muscles to decrease the action potential MF.

Another factor that may have influenced it is the type of muscle fatigue analysis used in this study, as it was based on the simple behavior of the electromyographic MF signal ${ }^{22}$. The international literature ${ }^{23}$ increasingly points to different approaches to muscle fatigue analysis, still trying to find which index best describes this phenomenon.

\section{CONCLUSION}

The effect of two TC approaches in young women was compared and no difference in their influence on the electrical activity of the suprahyoid musculature was found. Further studies approaching these results more in-depth, controlling other variables, such as the percentage of TC load, and having equal group sizes should be conducted.

\section{REFERENCES}

1. Gallerano G, Ruoppolo G, Silvestri A. Myofunctional and speech rehabilitation after orthodontic-surgical treatment of dento-maxillofacial dysgnathia. Prog Orthod. 2012;13(1):57-68.

2. Corrêa CC, Berretin-Felix G. Myofunctional therapy applied to upper airway resistance syndrome: a case report. CoDAS. 2015;27(6):604-9.

3. Rahal A. Exercicios miofuncionais orofaciais. In: Motta AR, Furlan RMMM, Tessitore A, Cunha DA, Berretin-Felix G, Silva $\mathrm{HJ}$ et al, editors. Motricidade orofacial: a atuação nos diferentes níveis de atenção à saúde. São José dos Campos, SP: Pulso Editorial, 2017. p.71-5.
4. Silva HJ, Cunha DA. A utilização de exercícios na terapia miofuncional orofacial. In: Motta AR, Furlan RMMM, Tessitore A, Cunha DA, Berretin-Felix $\mathrm{G}$, Silva $\mathrm{HJ}$ et al, editors. Motricidade orofacial: a atuação nos diferentes níveis de atenção à saúde. São José dos Campos, SP: Pulso Editorial, 2017. p.77-82.

5. Zemlin W. Princípios de anatomia e fisiologia em Fonoaudiologia. 4aed. Porto Alegre: Artes Médicas; 2000.

6. Rezende BA, Furlan RMMM, Casas EBL, Motta AR. Clinical assessment of the tongue in healthy young adults. Rev. CEFAC. 2016;18(3):559-67.

7. Reis VS, Araújo TG, Furlan RMMM, Motta AR. Correlation between tongue pressure and electrical activity of the suprahyoid muscles. Rev. CEFAC. 2017;19(6):792-800.

8. Verma RK, Johnson JJR, Goval M, Banumathy N, Goswami U, Panda NK. Oropharyngeal exercises in the treatment of obstructive sleep apnea: our experience. Sleep Breath. 2016;20(4):1193-201.

9. De Felício CM, Dias FVS, Trawitzki, LVV. Obstructive sleep apnea: focus on myofunctional therapy. Nat Sci Sleep. 2018;(10):271-86.

10. Ertuk N, Calik-Kutukcu E, Arikan H, Savci S, Inal-Ince D, Caliskan $\mathrm{H}$ et al. The effectiveness of oropharyngeal exercises compared to inspiratory muscle training in obstructive sleep apnea: a randomized controlled trial. Heart \& Lung. 2020;49(6):940-8.

11. Silva LK. Efetividade de um programa de terapia miofuncional no tratamento de respiração oral em indivíduos com má oclusão dentária: ensaio clínico [dissertation]. São Paulo (SP): Universidade de São Paulo, Faculdade de Odontologia de Bauru; 2017.

12. Ferreira KRG, Cunha DA, Albuquerque LCA, Silva HJ. Relationship between the electrical activity of suprahyoid musculature and tongue pressure during swallowing in the elderly. Rev. CEFAC. 2020;22(2):1-6.

13. Steele CM, Bayley MT, Peladeau-Pigeon M, Nagy A, Namasivayam AM, Stokely SL et al. A randomized trial comparing two tongue-pressure resistance training protocols for post-stroke dysphagia. Dysphagia. 2016;31(3):452-61.

14. Kim HD, Choi JB, Yoo SJ, Chang MY, Lee SW, Park JS. Tongue-to-palate resistance training improves tongue strength and oropharyngeal swallowing function in subacute stroke survivors with dysphagia. J Oral Rehabil. 2017;44(1):59-64. 
15. Wakabayashi $H$, Matsushima M, Momosaki R, Yoshida S, Mutai R, Yodoshi T et al. The effects of resistance training of swallowing muscles on dysphagia in older people: a cluster, randomized, controlled trial. Nutrition. 2018;(48):111-6.

16. Namiki C, Hara K, Tohara H, Kobayashi K, Chantaramanee A, Nakagawa $\mathrm{K}$ et al. Tonguepressure resistance training improves tongue and suprahyoid muscle functions simultaneously. Clinical Interventions in Aging. 2019;(14):601-8.

17. Park S, Cho JY, Lee BJ, Hwang J-M, Lee M, Hwang SY et al. Effects of the submandibular push exercise using visual feedback from pressure sensor: an electromyography study. Sci Rep. 2020;10(1):11772.

18. Batista DPF, Bagarollo MF. Surface electromyography in orofacial and cervical musculature in mouth breathing children: an integrative literature review. Rev. CEFAC. 2019;22(1):1-12.

19. Freitas GS, Mituuti CT, Furkim AM, BusanelloStella AR, Stefani FM, Arone MMAS et al. Electromyography biofeedback in the treatment of neurogenic orofacial disorders: systematic review of the literature. Audiol Commun Res. 2016;(21):1-10.

20. Furlan RMMM, Rezende BA, Motta AR. Comparison of the electric activity of the suprahyoid muscles during different lingual exercises. Audiol Commun Res. 2015;20(3):203-9.

21. Gazzoni M, Botter A, Vieira T. Surface EMG and muscle fatigue: multi-channel approaches to the study of myoelectric manifestations of muscle fatigue. Physiol. Meas. 2017;38(5):27-60.

22. Arnoni VW, Vasconcelos PB, Sousa LG, Ferreira B, Palinkas M, Righetti MA et al. Evaluation of the electromyographic fatigue of the masseter and temporalis muscles in individuals with osteoporosis. CRANIO®. 2018;37(4):254-63.

23. Gawda P, Ginszt M, Ginszt A, Pawlak H, Majcher P. Differences in myoelectric manifestations of fatigue during isometric muscle actions. Ann Agric Environ Med. 2018;25(2):296-9.

24. Coutrin GC, Guedes LU, Motta AR. Treinamento muscular na face. Rev Soc Bras Fonoaudiol. 2008;13(2):127-35.

25. Migliorucci RR, Passos DCBOF, Berretin-Felix G. Orofacial myofunctional therapy program for individuals undergoing orthognathic surgery. Rev. CEFAC. 2017;19(2):277-88.
26. Sekihata S, lida T, Honki H, Ikuta M, Komiyama O. Long-term Tongue Lift Training effects on tongue function. Int J Oral-Med Sci. 2020;18(3-4):325-31.

27. Larsson L, Degens H, Li M, Salviati L, Lee YI, Thompson $\mathrm{W}$ et al. Sarcopenia: aging-related loss of muscle mass and function. Physiol Rev. 2019;99(1):427-511.

28. Rezende FAC, Rosado LEFPL, Franceschinni SCC, Rosado G, Ribeiro RCL. Aplicabilidade do Índice de Massa Corporal na avaliação da gordura corporal. Rev Bras Med Esporte. 2010;16(2):90-4.

29. World Health Organization (WHO). Obesity: preventing and managing the global epidemic. Report of a WHO consultation on obesity. Geneva: WHO; 1998.

30. Genaro KF, Berretin-Felix G, Rehder MIBC, Marchesan IQ. Avaliação Miofuncional Orofacial Protocolo MBGR. Rev. CEFAC. 2009;11(2):237-55.

31. Folha GA. Ampliação das escalas numéricas do Protocolo Avaliação Miofuncional Orofacial (AMIOFE), validação e confiabilidade [thesis]. São Paulo (SP): Universidade de São Paulo, Faculdade de Medicina da Universidade de São Paulo; 2010.

32. Prates LS, Gois M, Berwig LC, Blanco-Dutra AP, Busanello-Stella AR, Silva AMT. Clinical and electromyographic evaluation of mastication within different facial growth patterns. Rev. CEFAC. 2016;18(1):104-12.

33. Silva AP, Carvalho ARR, Sassi FC, Silva MAA. The taping method effects on the trapezius muscle in healthy adults. CoDAS. 2019;31(5):1-8.

34. Santos AC, Silva CAB. Surface electromyography of masseter and temporal muscles with use percentage while chewing on candidates for gastroplasty. ABCD Arq Bras Cir Dig. 2016;29(1):48-52.

35. Torres GMX, César CPHAR. Physiology of exercise in orofacial motricity: knowledge about the issue. Rev. CEFAC. 2019;21(1):1-10.

36. McKenna VS, Zhang B, Haines MB, Kelchner LN. A systematic review of isometric lingual strength-training programs in adults with and without dysphagia. Am J Speech Lang Pathol. 2017;26(2):524-39.

37. Van den Steen L, Vanderwegen J, Guns C, Elen R, Bodt M, Nuffelen GV. Tongue-strengthening exercises in healthy older adults: does exercise load matter? A randomized controlled trial. Dysphagia. 2019;34(3):315-24. 